\title{
The use of radiofrequency ablation in pancreatic cancer in the midst of the dawn of immuno-oncology
}

\author{
Isabella Reccia ${ }^{1}$ (D) Jayant Kumar ${ }^{1} \cdot$ Nagy Habib $^{1} \cdot$ Mikael Sodergren $^{1}$
}

Received: 1 September 2018 / Accepted: 26 September 2018 / Published online: 4 October 2018

๑) Springer Science+Business Media, LLC, part of Springer Nature 2018

\begin{abstract}
Despite significant improvement in treatment, the prognosis of pancreatic ductal adenocarcinoma remains poor as the biology of the tumour affects survival even when a radical resection has been performed. Pancreatic cancer remains resistant to currently available chemotherapeutic options. Recently, immunotherapy has achieved significant results in certain types of cancer. However, for pancreatic cancer, results were not initially encouraging as pancreatic cancer microenvironment is highly immunosuppressive. Radiofrequency ablation is currently used as treatment option especially for liver cancer with significant results. However, in pancreatic cancer, the use of radiofrequency ablation is relatively new. Radiofrequency ablation has been identified as a promising mechanism to induce antigen-presenting cell infiltration and enhance systemic antitumour T-cell immune response and tumour regression. In this short communication, we briefly review the role of radiofrequency ablation in pancreatic cancer and explore the idea that the combination of radiofrequency ablation with immunotherapy could represent a novel and promising treatment.
\end{abstract}

Keywords Pancreatic cancer $\cdot$ Immunotherapy $\cdot$ Radiofrequency ablation $\cdot$ Survival

$\begin{array}{ll}\text { Abbreviations } \\ \text { CTLA4 } & \text { Anti-cytotoxic T-lymphocyte antigen } 4 \\ \text { Teffs } & \text { Effector T cells } \\ \text { GM-CSF } & \begin{array}{l}\text { Granulocyte-macrophage colony-stimulating } \\ \text { factor }\end{array} \\ \text { PDAC } & \begin{array}{l}\text { Pancreatic ductal adenocarcinoma } \\ \text { PD1 }\end{array} \\ \text { Programmed cell death protein 1 } \\ \text { RFA } & \text { Radiofrequency ablation } \\ \text { Tregs } & \text { Regulatory T cells }\end{array}$

Pancreatic ductal adenocarcinoma (PDAC) remains one of the most deadly forms of cancer and compared with other solid organ malignancies, significant improvements in survival have not been evident over the last few decades.

Isabella Reccia

isabella.reccia@gmail.com

Mikael Sodergren

m.sodergren@imperial.ac.uk

Nagy Habib

nagy.habib@imperial.ac.uk

1 Department of Surgery \& Cancer, Hammersmith Campus, Imperial College London, Du Cane Road, London W12 0NN, UK
Radical surgery remains the only curative option; however, only $15 \%$ of patients are suitable for surgical resection at presentation, and patients with stage III and IV unresectable diseases receiving chemotherapy have median survival of 11.4 and 6 months, respectively. Advances in surgical techniques to achieve a negative margin including vascular resection and reconstruction have allowed borderline operable patients to gain similar, although limited, survival advantages to patients who present with no vascular involvement with a median survival of 18 months [1]. In spite of radical surgery, the biology of the tumour can affect survival even when a $\mathrm{R} 0$ resection has been performed.

Following the publication of the ESPAC-4 trial, adjuvant treatment with gemcitabine and capecitabine should be considered the gold standard for resectable disease; however, the prognosis remains bleak for the majority of patients with a median survival of 28 months [2]. Despite recent excitement surrounding the use of neoadjuvant FOLFIRINOX to downstage borderline resectable patients, survival data confirm PDAC to be relatively resistant to currently available chemotherapeutic options. The requirement for an effective systemic treatment to this aggressive disease is urgent.

Focus on immunotherapy has again come to the fore following the publication of impressive response and survival outcomes in melanoma and lung cancer. Unfortunately, the 
use of checkpoint inhibitors with programmed cell death protein 1 (PD1) antibodies and anti-cytotoxic T-lymphocyte antigen 4 (CTLA4) antibodies in pancreatic cancer has been disappointing. This has been attributed partly to the lack of effector CD8+ T cells and CD103+ dendritic cells and excess of myeloid-derived suppressor cells. The question is therefore why checkpoint inhibitors are more effective in some solid organ malignancies than others. The principal characteristic of the pancreatic cancer microenvironment is that it is highly immunosuppressive with only a limited neoantigen-presenting capacity. The tumour and associated stromal cells abort T-cell responses through apoptosis, dysfunction, and ineffective targeting. Therefore, simply the recruitment of more $\mathrm{T}$ cells through checkpoint inhibitors appears relatively ineffective in the context of this tumour microenvironment. Tilting the immunobalance of the patient can be accomplished in several ways, including the use of vaccines, for example, GVAX, which consists of patient-specific pancreatic cancer cells genetically modified to secrete cytokine granulocyte-macrophage colony-stimulating factor (GM-CSF) that could induce immunostimulation and have an antineoplastic effect [3].

Radiofrequency ablation (RFA) has been identified as a promising mechanism to induce antigen-presenting cell infiltration and enhance systemic antitumour T-cell immune response and tumour regression. Thermal ablation is followed by a marked local inflammatory response with a dense T-cell infiltrate with data suggesting that the heat shock delivery and massive necrotic cell death by RFA lead to immune activation and presentation of otherwise cryptic antigens, thus inducing a tumour-specific T-cell response $[4,5]$.

The use of RFA in PDAC has been successfully explored with positive impact on survival with acceptable safety profile [6]. In a small series where RFA was applied to inoperable PDAC at laparotomy followed by bypass, it was observed that RFA could achieve local control in large tumours and regression of small tumours with subsequent benefits in survival and quality of life [7]. Further data in stage III unresectable locally advanced pancreatic cancer have shown median survival following RFA ranging from 20 to 33 months [8]. Application of RFA to the pancreas has been recently shown to be feasible with similar clinical outcomes using both the percutaneous and endoscopic ultrasound routes. Moreover, RFA has shown survival benefit when applied endoscopically in PDAC with biliary obstruction followed by metal stent insertion and to unblock an occluded stent [9].

Although RFA modulates both adaptive and innate immunity, the response may be insufficient on its own to induce complete tumour destruction and also to provide a durable effect. Thus, a combination of RFA and immunomodulating drugs in the treatment of solid tumours may be effective [10]. The microenvironment in PDAC is particularly important, with macrophages, myeloid-derived suppressor cells and regulatory T cells (Tregs) involved in immunosuppressive antitumour activity. It has been shown that PDAC is associated with an increased number of Tregs and inactivated effector T cells (Teffs). Bassi et al. have recently demonstrated that RFA in PDAC leads to early significant increase of IL-6 proinflammatory chemokine with lack of activation of immunosuppressive lymphocytes (CD4+CD25+Foxp3+regulatory T cells), monocytes and plasmocytoid dendritic cells [11]. Giardino at al. observed a general activation of an adaptive response along with a decrease of immunosuppression that was evident even a few weeks after open ablation in ten patients with locally advanced PDAC [12].

In summary, conceptually, the combination of RFA with immunotherapy represents a novel angle of attack against a malignancy that we have not yet been able to beat.

Author contributions

IR: planning the study, collecting and interpreting data, and drafting the manuscript. MS: collecting and interpreting data, and drafting the manuscript. NH: revising it critically for important intellectual content.

\section{Compliance with ethical standards}

Conflict of interest The authors report no conflicts of interest, but Nagy Habib is a shareholder and director of EMcision Limited, which has developed the Habib 4X, an RFA device, not manufactured or currently marketed by this company.

\section{References}

1. Ravikumar R, Sabin C, Abu Hilal M, Bramhall S, White S, Wigmore $S$, et al. Portal vein resection in borderline resectable pancreatic cancer: a United Kingdom multicenter study. J Am Coll Surg. 2014;218(3):401-11.

2. Neoptolemos JP, Palmer DH, Ghaneh P, Psarelli EE, Valle JW, Halloran CM, et al. Comparison of adjuvant gemcitabine and capecitabine with gemcitabine monotherapy in patients with resected pancreatic cancer (ESPAC-4): a multicentre, open-label, randomised, phase 3 trial. Lancet. 2017;389(10073):1011-24.

3. Salman B, Zhou D, Jaffee EM, Edil BH, Zheng L. Vaccine therapy for pancreatic cancer. Oncoimmunology. 2013;2(12):e26662.

4. Dromi SA, Walsh MP, Herby S, Traughber B, Xie J, Sharma KV, et al. Radiofrequency ablation induces antigen-presenting cell infiltration and amplification of weak tumor-induced immunity. Radiology. 2009;251(1):58-66.

5. Zerbini A, Pilli M, Penna A, Pelosi G, Schianchi C, Molinari A, et al. Radiofrequency thermal ablation of hepatocellular carcinoma liver nodules can activate and enhance tumor-specific T-cell responses. Cancer Res. 2006;66(2):1139-46.

6. Fegrachi S, Besselink MG, van Santvoort HC, van Hillegersberg R, Molenaar IQ. Radiofrequency ablation for unresectable locally advanced pancreatic cancer: a systematic review. HPB (Oxford). 2014;16(2):119-23. 
7. Hadjicostas P, Malakounides N, Varianos C, Kitiris E, Lerni F, Symeonides P. Radiofrequency ablation in pancreatic cancer. HPB (Oxford). 2006;8(1):61-4.

8. Girelli R, Giardino A, Frigerio I, et al. Survival after radiofrequency of stage III pancreatic carcinoma: a wind of change? HPB (Oxford) 2011;13(Suppl.2):15

9. Kallis Y, Phillips N, Steel A, Kaltsidis H, Vlavianos P, Habib N, et al. Analysis of endoscopic radiofrequency ablation of biliary malignant strictures in pancreatic cancer suggests potential survival benefit. Dig Dis Sci. 2015;60(11):3449-55.
10. Haen SP, Pereira PL, Salih HR, Rammensee HG, Gouttefangeas C. More than just tumor destruction: immunomodulation by thermal ablation of cancer. Clin Dev Immunol. 2011;2011:160250.

11. Giardino A, Innamorati G, Girelli R, et al. Radiofrequency ablation of locally advanced pancreatic cancer: immunostimulation patterns. 12th Biennal E-AHPBA Congress. Mainz, 2017

12. Giardino A, Innamorati G, Ugel S, Perbellini O, Girelli R, Frigerio I, et al. Immunomodulation after radiofrequency ablation of locally advanced pancreatic cancer by monitoring the immune response in 10 patients. Pancreatology. 2017;17:962-6. 